\title{
Photoluminescence Spectroscopy of Silica-Based Mesoporous Materials
}

\author{
Yuri D. Glinka* \\ Institute of Atomic and Molecular Sciences, Academia Sinica, P.O. Box 23-166, Taipei 106, Taiwan, R.O.C.

\begin{abstract}
Sheng-Hsien Lin, Lian-Pin Hwang, and Yit-Tsong Chen
Institute of Atomic and Molecular Sciences, Academia Sinica, P.O. Box 23-166, Taipei 106, Taiwan, R.O.C., Department of Chemistry, National Taiwan University, Taipei 106, Taiwan, R.O.C.
\end{abstract}

Received: March 14, 2000; In Final Form: June 19, 2000

\begin{abstract}
The photoluminescence (PL) at 300 and $90 \mathrm{~K}$ from the mesoporous silicas (MSs) [ordered (MCM-41) and disordered (DMSs)] of variously sized pores induced either by $\operatorname{ArF}$ laser light $\left(\lambda_{\mathrm{exc}}=193 \mathrm{~nm}\right)$ or by $\mathrm{Nd}$ : YAG (yttrium-aluminum-garnet) laser light $\left(\lambda_{\mathrm{exc}}=266 \mathrm{~nm}\right)$ was studied. The spectra were measured in both conventional and time-resolved detection modes. It has been shown that PL induced by 266-nm light results from a direct one-photon excitation of hydrogen-related species (the green band) situated on the internal pore surfaces and nonbridging oxygen hole centers (NBOHCs, the red bands) located both on the surface and inside $\sim 1$-nm sized bulk fragments (pore walls). Alternatively, an indirect excitation of the light emitters due to the energy transfer through two-photon-produced free excitons (FEs) is responsible for PL in the case of 193-nm excitation. In addition, the self-trapping of FEs followed by radiative relaxation results in the blue band, which can only be induced by 193-nm light. The PL properties of MSs in comparison with those of silica nanoparticles and bulk silica are discussed.
\end{abstract}

\section{Introduction}

In the past few years silica-based mesostructures gained enormous attention since they found great utility as catalysts and sorption media with a large internal surface area. ${ }^{1,2}$ The mesostructures can be divided into two different groups. One of them includes the disordered materials. The composites of silica nanoparticles with the primary particle size of $10-50 \mathrm{~nm}$ are an example of disordered mesostructures. ${ }^{3-5}$ Composites usually show arbitrary shape and look like fractal-type aggregates. As this takes place, smaller size particles can form the weblike structures. ${ }^{6}$ Recently discovered silica-based mesoporous molecular sieves can occur as a disordered form, well as an ordered one. ${ }^{1}$ Therefore, the second group involves the ordered mesoporous molecular sieves, designated MCM-41. In either case the materials are characterized by porosity and are usually called mesoporous silicas (MSs). The pore diameters for MSs ranges from 2 to $10 \mathrm{~nm}^{1,2}$ and the specific surface area is $2-3$-fold larger as compared to nanoparticle composites, ${ }^{1-4}$ indicating that the ordered and disordered MSs (MCM-41 and DMSs, respectively) improve significantly the adsorption properties of silica-based mesostructures.

Up to now, the nature of walls among pores in MSs is not fully understood. Such information could be obtained from spectroscopic measurements. However, these types of experimental works are few in number so far. Meanwhile, MSs resemble the structure of the ropes of carbon nanotubes. ${ }^{7,8}$ In both cases the materials are characterized by distributed inorganic nanochannels, the dimensions of which can vary. It is expected that such materials can find numerous applications in nanoscale technology.

* Permanent address of corresponding author: Institute of Surface Chemistry of the National Academy of Sciences of Ukraine, prospekt Nauki 31, Kiev 252650, Ukraine. E-mail: glinka@po.iams.sinica.edu.tw.
Particular emphasis for MSs has been placed on the transmission electron microscopy, ${ }^{1,2}$ adsorption-desorption measurements, ${ }^{1,2,9}{ }^{1} \mathrm{H}$ nuclear magnetic resonance spectroscopy, ${ }^{2} \mathrm{X}$-ray diffraction (XRD), ${ }^{1,2,10}$ and the infrared spectra of $\equiv \mathrm{Si}-\mathrm{OH}$ and $\equiv \mathrm{Si}-\mathrm{H}$ species located on the pore internal surface. ${ }^{9,11,12}$ Because the photoluminescence (PL) from bulk $\mathrm{SiO}_{2}$ polymorphs is quite well-studied, ${ }^{13-27}$ the comparative PL investigation of MSs could also give useful information about the properties of new materials synthesized. To our knowledge, such consideration has not been done before. Moreover, the recent study of $\mathrm{SiO}_{2}$ thin films and nanoparticles showed that the PL properties of nanometer-sized objects ${ }^{28-33}$ are somewhat different from those of bulk materials. ${ }^{13-27}$ This fact is expected to be advantageous for the characterization of silica nanoscales and their applications. Hence, it is extremely important to probe the PL from MSs since the thickness of bulk fragments (pore walls) is normally $\sim 1 \mathrm{~nm} .{ }^{1,2}$

It is known that bulk $\mathrm{SiO}_{2}$ is a wide band gap insulator (the band gap of bulk silica $E_{\mathrm{g}} \cong 11 \mathrm{eV}$ ). ${ }^{13}$ As a result, energetic electrons or ultraviolet (UV) or vacuum UV light can only induce the intrinsic PL, occurring from electron-hole recombination in excitons. ${ }^{13,22-27}$ Moreover, it is necessary to use the multiphoton (MP) excitation in order to produce excitons in that material. ${ }^{24-27,33}$ It has been shown recently that there exist several pathways for two-photon (TP) -produced free exciton (FE) energy relaxation, including self-trapping followed by the self-trapped exciton (STE) PL (STEPL), the FE energy transfer to impurities and structural defects with their subsequent intrinsic PL, the nonradiative decay, and/or defect formation. ${ }^{33}$ From a physical point of view, it is interesting to compare the properties of bulk $\mathrm{SiO}_{2}$ and MSs, especially those that are related to the carrier and FE transport. The latter process is evidenced to be responsible for the formation of defects, ${ }^{26}$ that affect the optical and electrical properties of these materials. Because the process 
can involve the surface species as well, the FE relaxation can reconstruct the pore internal surface and hence affect the adsorption efficiency of MSs.

Some other type of PL from bulk $\mathrm{SiO}_{2}$ polymorphs is associated with structural defects, such as nonbridging oxygen hole centers $(\mathrm{NBOHCs}), \equiv \mathrm{Si}-\mathrm{O}^{\bullet}$, oxygen-deficient centers (oxygen vacancies, divacancies), peroxy linkages and radicals, dicoordinated silicon, and $E^{\prime}$ centers. The impurities, especially hydrogen ( $\equiv \mathrm{Si}-\mathrm{H}$ and $\equiv \mathrm{Si}-\mathrm{OH}$ groups), also dramatically control the PL properties of $\mathrm{SiO}_{2}$ materials. It is known that PL from defects and impurities can be induced directly by UV or visible light as a one-photon process. ${ }^{14-21}$ Accordingly, one can observe several PL bands resulting from the variety of PL centers present. Hence, the PL spectroscopy has great potential to reveal the structural peculiarities of MSs and impurities typically present in these materials.

Note that the spectroscopic properties that will be discussed for MSs are closely related to those of silicon-hydrogen alloy films and porous silicon. ${ }^{34-40}$ It is known that PL from these materials is the object of much concentrated attention for nanoscale electronics, ${ }^{34}$ but the origin of emission has not yet been completely clarified. Therefore, the study of MSs is also of profound importance for silicon-based electronic technology.

In the current paper we present the experimental study of PL from MSs induced either by $\mathrm{ArF}$ laser light $\left(\lambda_{\text {exc }}=193 \mathrm{~nm}\right)$ or by Nd:YAG (yttrium-aluminum - garnet) laser light $\left(\lambda_{\text {exc }}=266\right.$ $\mathrm{nm})$. The materials synthesized were examined by the powder XRD method. Accordingly, the MCM-41 and DMSs of variously sized pores were identified. The PL spectra were measured both in the conventional detection mode by a charge-coupled device (CCD) camera and in the time-resolved detection mode by a gated electronic system. Major attention is given to the effect of pore internal surface conditions, varying with heat pretreatment in air, onto the PL properties.

\section{Experimental Section}

2.1. Material Synthesis. Three MSs were used in the current study (No. 1-3). The materials have been synthesized using the following gel composition: 1.0:0.35:0.28:100 $\mathrm{SiO}_{2}: \mathrm{CnTAB}$ : $\mathrm{H}_{2} \mathrm{SO}_{4}: \mathrm{H}_{2} \mathrm{O} ;[\mathrm{CnTAB}=$ alkyl trimethylammonium bromide; $n$ $=10$ for sample 2 and $n=16$ for samples 1 and 3]. The synthesis of sample 1 was done as follows. Cetyltrimethylammonium bromide (CTAB) (Acros, 99\%) was dissolved in distilled water by stirring at $308 \mathrm{~K}$. The silica source, sodium silicate solution (Aldrich, $14 \% \mathrm{NaOH}$ and $27 \% \mathrm{SiO}_{2}$ ), was added to the solution under stirring for $20 \mathrm{~min}$. The $\mathrm{pH}$ of the solution mixture was adjusted to 10 by dropwise addition of $1.2 \mathrm{M}$ sulfuric acid (Acros, 95\%). The gel mixture was further stirred for $1 \mathrm{~h}$ and transferred to a Teflon-lined autoclave and then heated at $373 \mathrm{~K}$ for $48 \mathrm{~h}$. The product was obtained by filtration, washed with distilled water, dried in air at room temperature, and calcined at $833 \mathrm{~K}$ for $6 \mathrm{~h}$.

Synthesis of MSs 2 and 3 was also done by a similar procedure, but decyltrimethylammonium bromide (Acros, 99\%) was used as a template instead of CTAB for sample 2, while trimethyl benzene (TMB) (Acros, 97\%) (TMB/CTAB 1.95) was used as an additive for the synthesis of sample 3 with the aim of varying pore size. ${ }^{10}$

2.2. XRD Characterization. XRD data of the samples were collected by a Scientag $\mathrm{XI}$ diffractometer using $\mathrm{Cu}-\alpha$ radiation. The X-ray pattern of sample 1 matched well with the reported pattern for MCM-41. ${ }^{1,2}$ The X-ray patterns for samples 2 and 3 were similar to those reported earlier, ${ }^{10}$ being somewhat broad, suggesting the presence of disorder or variability in the structure.
TABLE 1: $d_{100}$ Spacings for Different MSs

\begin{tabular}{clc}
\hline sample no. & sample type & $d_{100} / \mathrm{nm}$ \\
\hline 1 & MCM-41 & 3.83 \\
2 & DMS & 2.9 \\
3 & DMS & 7.1
\end{tabular}

The $d_{100}$ spacing (repeat distance) is found to be less for sample 2 than that for MCM-41 (sample 1), because a smaller surfactant, decyltrimethylammonium bromide, was used instead of CTAB, while the $d_{100}$ spacing for sample 3 is enhanced due to a swelling effect of the TMB molecules during synthesis. The resulting $d_{100}$ spacings corresponding to the reflection peak are listed in Table 1 . On the basis of XRD patterns and previous data, ${ }^{1,2,10}$ we have concluded that sample 1 is characterized by the crystalline ordering (MCM-41). Samples 2 and 3 were found to be amorphous (DMSs). Since $d_{100}$ spacings actually reflect pore sizes, neglecting the wall thickness $(0.8-1.2 \mathrm{~nm}),{ }^{1,2}$ we will associate the $d_{100}$ spacings of MSs with their pore sizes. The powders were pressed into pellets and then heat pretreated in air for $2 \mathrm{~h}$ at various temperatures $T_{\mathrm{ht}}=300$ (initial sample), 873, and $1173 \mathrm{~K}$.

2.3. PL Measurements. The PL measurements were performed in a vacuum chamber at $T=300$ and $90 \mathrm{~K}$ using $\mathrm{ArF}$ pulsed laser $\left[\lambda_{\text {exc }}=193 \mathrm{~nm}(20 \mathrm{~ns})\right.$; Lumonics, EX-742] with a repetition rate of $10 \mathrm{~Hz}$ or Nd:YAG pulsed laser $\left[\lambda_{\text {exc }}=266\right.$ $\mathrm{nm}$ (8 ns); Spectra Physics, GCR-190] with a repetition rate of $30 \mathrm{~Hz}$. The laser beams were focused by a $30-\mathrm{cm}$ lens into a $0.03 \mathrm{~cm}^{2}$ spot. The laser light intensity could be varied by a set of quartz plates. The sample was oriented to the laser beam by $45^{\circ}$. The PL was collected in a conventional $90^{\circ}$ geometry by a 0.5-m SpectraPro-500 monochromator (Acton Research Corporation) with 1200 grooves $/ \mathrm{mm}$ grating blazed for the 500 $\mathrm{nm}$. The spectra were recorded using a CCD camera (Princeton Instruments, $330(\times 1100$ pixels $)$ within an accumulation time of $2 \mathrm{~s}$ (the conventional detection mode) or an R943-02 photomultiplier (peak wavelength $300-800 \mathrm{~nm}$; Hamamatsu) connected with a gated electronic system (SRS-250, Stanford Research System) in time-resolved scans. The data acquisition is processed in a computer equipped by CSMA software (Princeton Instruments). A set of color filters has been used in order to cut stray radiation from the lasers and scattered laser light from the specimens. The intensity dependences of the PL yield (IDPLY) were measured using the CCD camera with an accumulation time of $10 \mathrm{~s}$ applied for every laser light intensity. A LeCroy oscilloscope is used to measure the PL time decay.

\section{Results and Discussion}

The $T_{\mathrm{ht}}$ dynamics of PL induced by ArF or Nd:YAG laser light and measured in the conventional detection mode for MSs with variously sized pores is shown in Figures $1-3$. Note first that the spectra are similar to those obtained for a silica nanoparticle. . $^{32,33}$ Accordingly, one can observe three bands located in the blue, green, and red spectral ranges. The green PL band is peaked at $\sim 2.37 \mathrm{eV}$ and shows a progression with spacings of about $630 \mathrm{~cm}^{-1}$ for the initial and heat-pretreated at $T_{\mathrm{ht}}=873 \mathrm{~K}$ specimens. The same type of PL has been assigned to hydrogen-related species located on the silica nanoparticle surface. ${ }^{32,33}$ Totally, it is reasonable to expect that the PL band assignment made for the nanoparticles will also be applicable for MSs. Therefore, the 2.37-eV band for MSs can be assigned to hydrogen-related species located on the pore internal surfaces. The progression is due to the electronvibrational coupling involving bending vibrations $\equiv \mathrm{Si}-\mathrm{H}$ in the ground electronic state. ${ }^{32}$ The heat pretreatment dramatically 


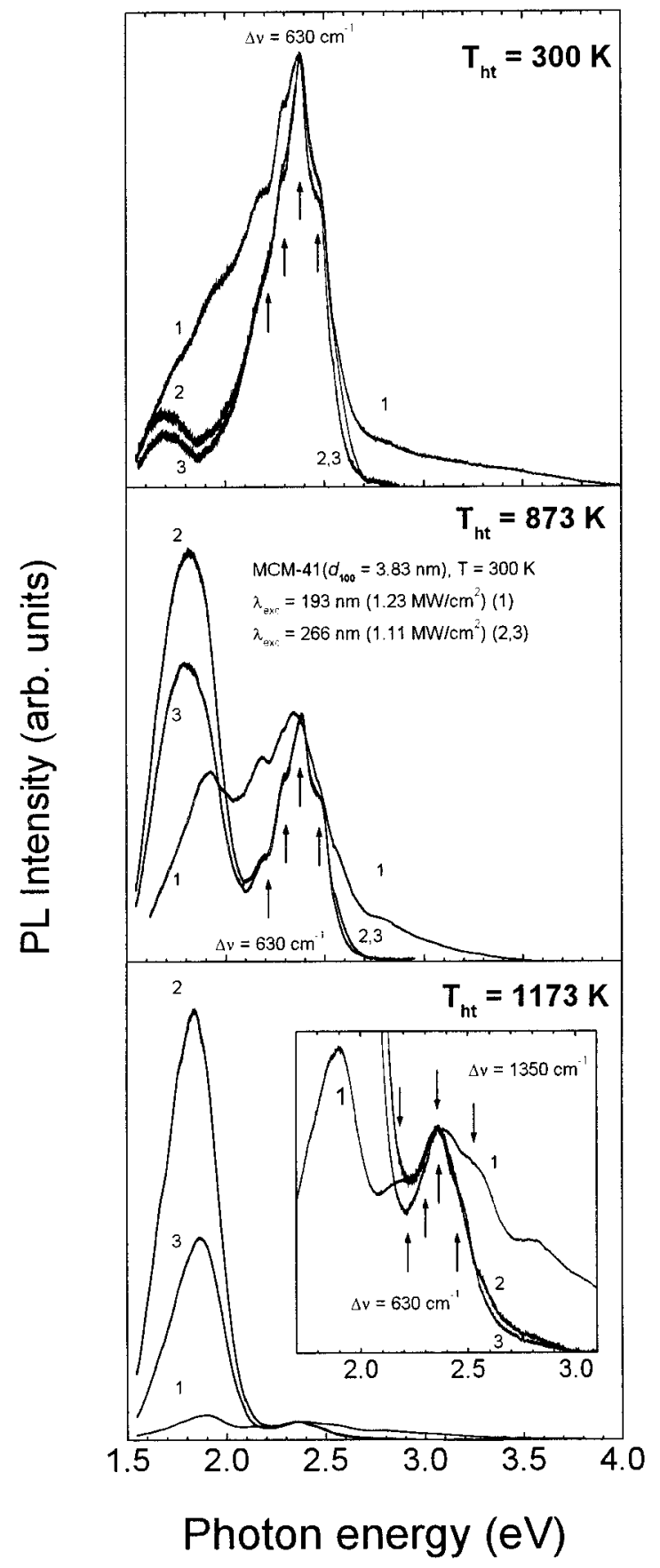

Figure 1. The $T_{\text {ht }}$ dynamics of PL spectra measured at $300 \mathrm{~K}$ by CCD camera for MCM-41 $\left(d_{100}=3.83 \mathrm{~nm}\right)$. The spectra were normalized at the $2.37-\mathrm{eV}$ band intensity; $\lambda_{\mathrm{exc}}=193 \mathrm{~nm}, I_{\mathrm{L}}=1.23 \mathrm{MW} / \mathrm{cm}^{2}(1)$; $\lambda_{\text {exc }}=266 \mathrm{~nm}, I_{\mathrm{L}}=1.11 \mathrm{MW} / \mathrm{cm}^{2}(2,3)$. Curves 2 and 3 correspond to the nonirradiated and preirradiated with $\lambda_{\mathrm{exc}}=193 \mathrm{~nm}\left(I_{\mathrm{L}}=1.23\right.$ $\mathrm{MW} / \mathrm{cm}^{2}$ ) specimens, respectively. The vibrational progression spacings $(\Delta v)$ are shown by arrows. The inset shows the same PL spectra on an enlarged scale.

decreases the PL band intensity because of hydrogen removal ${ }^{5}$

$$
(\equiv \mathrm{Si}-\mathrm{H}) \stackrel{T_{\mathrm{ht}} \geq 873 \mathrm{~K}}{\longrightarrow}\left(\equiv \mathrm{Si} i^{\bullet}\right)+\mathrm{H}^{0 \uparrow}
$$

At the same time, the green PL band intensity did not vary within the given time of laser irradiation, so it has been selected to normalize the intensity of spectra taken under different experimental conditions (Figures 1-3). However, the PL efficiency as a whole was much higher in the case of 193-nm excitation compared to that of the 266-nm one, despite the fact that the similar laser light intensities were applied.

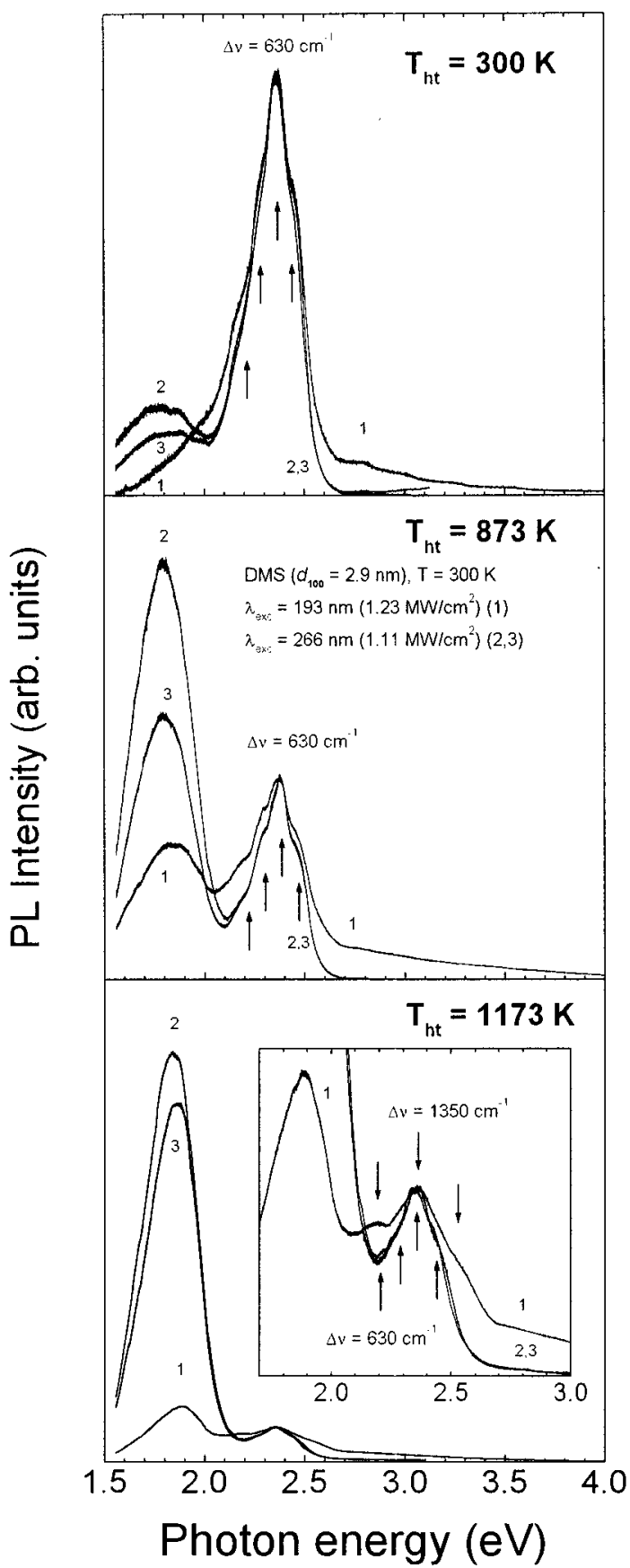

Figure 2. The $T_{\text {ht }}$ dynamics of PL spectra measured at $300 \mathrm{~K}$ by CCD camera for DMS $\left(d_{100}=2.9 \mathrm{~nm}\right)$. The spectra were normalized at the $2.37-\mathrm{eV}$ band intensity; $\lambda_{\mathrm{exc}}=193 \mathrm{~nm}, I_{\mathrm{L}}=1.23 \mathrm{MW} / \mathrm{cm}^{2}(1) ; \lambda_{\mathrm{exc}}=$ $266 \mathrm{~nm}, I_{\mathrm{L}}=1.11 \mathrm{MW} / \mathrm{cm}^{2}(2,3)$. Curves 2 and 3 correspond to the nonirradiated and preirradiated with $\lambda_{\text {exc }}=193 \mathrm{~nm}\left(I_{\mathrm{L}}=1.23 \mathrm{MW} /\right.$ $\left.\mathrm{cm}^{2}\right)$ specimens, respectively. The vibrational progression spacings $(\Delta v)$ are shown by arrows. The inset shows the same PL spectra on an enlarged scale.

The heat pretreatment also initiates the condensation of surface hydroxyls leading to NBOHC formation, ${ }^{4}$

$$
\begin{gathered}
2(\equiv \mathrm{Si}-\mathrm{OH}) \stackrel{T_{\mathrm{ht}} \geq 873 \mathrm{~K}}{\longrightarrow}\left(\equiv \mathrm{Si}-\mathrm{O}^{\bullet}\right)+(\equiv \mathrm{Si})+\mathrm{H}_{2} \mathrm{O}^{\uparrow} \\
2(\equiv \mathrm{Si}-\mathrm{OH}) \stackrel{T_{\mathrm{ht}} \geq 873 \mathrm{~K}}{\longrightarrow}\left(\equiv \mathrm{Si}-\mathrm{O}^{\circ} \mathrm{O}-\mathrm{Si} \equiv\right)+\mathrm{H}_{2} \uparrow
\end{gathered}
$$

As this takes place, the progression spacing increases up to $1350 \mathrm{~cm}^{-1}$ in spectra taken with $193-\mathrm{nm}$ excitation when specimens were heat-pretreated at $T_{\mathrm{ht}}=1173 \mathrm{~K}$. We explain 


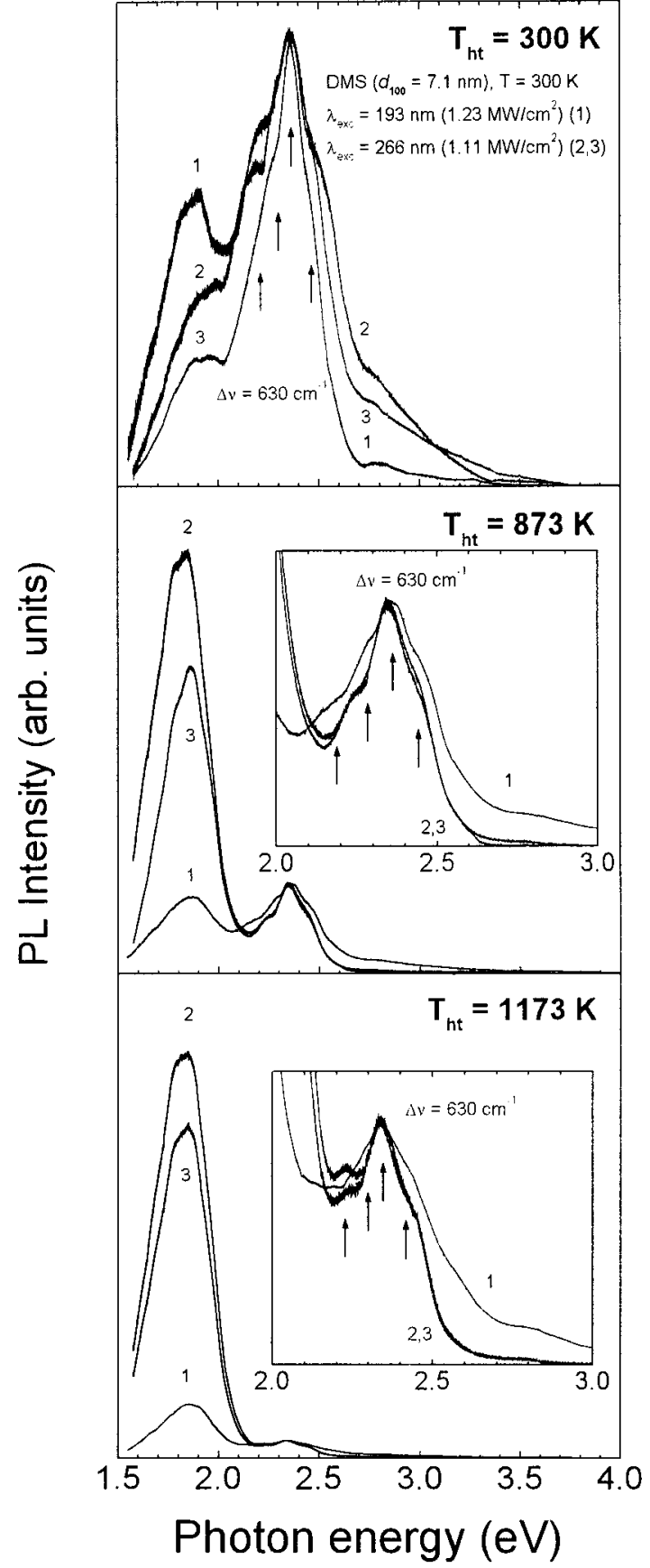

Figure 3. The $T_{\mathrm{ht}}$ dynamics of PL spectra measured at $300 \mathrm{~K}$ by CCD camera for DMS $\left(d_{100}=7.1 \mathrm{~nm}\right)$. The spectra were normalized at the $2.37-\mathrm{eV}$ band intensity; $\lambda_{\mathrm{exc}}=193 \mathrm{~nm}, I_{\mathrm{L}}=1.23 \mathrm{MW} / \mathrm{cm}^{2}(1) ; \lambda_{\mathrm{exc}}=$ $266 \mathrm{~nm}, I_{\mathrm{L}}=1.11 \mathrm{MW} / \mathrm{cm}^{2}(2,3)$. Curves 2 and 3 correspond to the nonirradiated and preirradiated with $\lambda_{\text {exc }}=193 \mathrm{~nm}\left(I_{\mathrm{L}}=1.23 \mathrm{MW} /\right.$ $\left.\mathrm{cm}^{2}\right)$ specimens, respectively. The vibrational progression spacings $(\Delta v)$ are shown by arrows. Insets show the same PL spectra on an enlarged scale.

this feature as resulting from interaction between $\equiv \mathrm{Si}-\mathrm{H}$ and nearest NBOHCs. It has been suggested for silica nanoparticles that the spacing increase is due to the interfacial water formation from $\equiv \mathrm{Si}-\mathrm{O}^{\bullet}$ and $\equiv \mathrm{Si}-\mathrm{H}$ species when the concentration of NBOHCs becomes large enough. ${ }^{32}$ The resulting frequency corresponds to $\mathrm{OH}$ bending vibrations in interfacial water approaching the frequency of gas-phase $\mathrm{H}_{2} \mathrm{O}\left(\sim 1595 \mathrm{~cm}^{-1}\right){ }^{41}$ Hence, we conclude that similarly bonded water-like species form in MSs as well. However, they are expected to occur inside pores. To comply with stoichiometric requirements, we assume that $\equiv \mathrm{Si}-\mathrm{O}^{\bullet}$ and $\equiv \mathrm{Si}-\mathrm{H}$ are placed nearby pore hexagon sides.

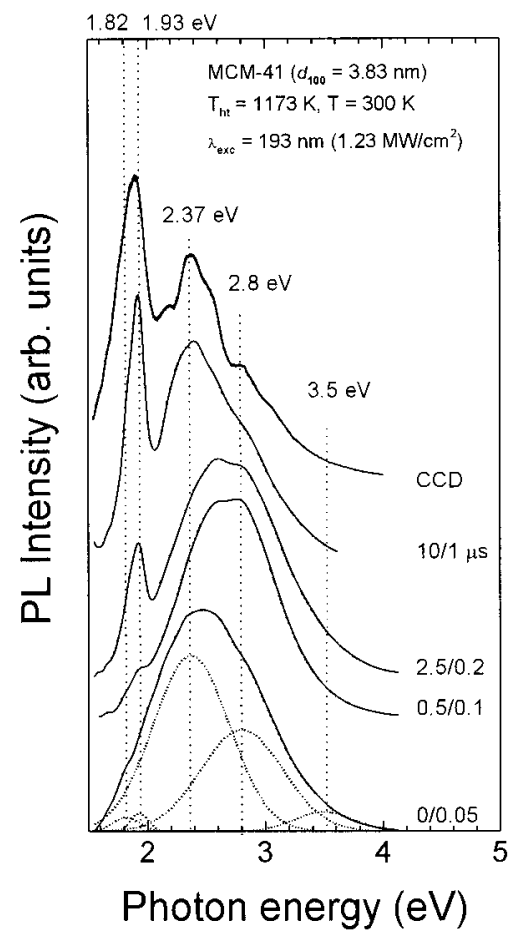

Figure 4. Time-resolved PL spectra measured at $300 \mathrm{~K}$ for MCM-41 $\left(d_{100}=3.83 \mathrm{~nm}\right)$ heat-pretreated at $T_{\mathrm{ht}}=1173 \mathrm{~K}$ in comparison to that taken by CCD camera. The spectra were normalized through the bluegreen band maximum; $\lambda_{\text {exc }}=193 \mathrm{~nm}, I_{\mathrm{L}}=1.23 \mathrm{MW} / \mathrm{cm}^{2}$. The individual spectrum baselines are arbitrarily shifted for better observation. Numbers at the right side indicate the gate delay and gate width (in microseconds), respectively, at which the corresponding timeresolved spectrum was measured. Dashed lines are Gaussian profiles corresponding to different PL bands. Dashed vertical lines and corresponding numbers in electronvolts show the positions of Gaussian component peaks. The spectrum measured by CCD camera repeats the corresponding curve in Figure 1.

Note also that bonded water-like species appear only after a high-temperature pretreatment $\left(T_{\mathrm{ht}}=1173 \mathrm{~K}\right)$ and the maximal rate occurs for MCM-41, likely to indicate the most suitable pore size at which such species can be formed (Figures 1-3). By contrast, the progression spacing remains to be the same $\left(630 \mathrm{~cm}^{-1}\right)$ for all spectra taken from specimens heat-pretreated at various $T_{\mathrm{ht}}$ when $266-\mathrm{nm}$ light was applied, showing that the isolated terminal $\equiv \mathrm{Si}-\mathrm{H}$ can only be excited in this case.

There exist two PL bands in the red range peaking at $\sim 1.9$ and $\sim 1.8 \mathrm{eV}$, as for silica nanoparticles. The bands were suggested to originate from bulk and surface NBOHCs, respectively. ${ }^{32,33}$ Because the band intensities increase dramatically with $T_{\mathrm{ht}}$, in accordance with reactions 2 and 3 (Figures $1-3$ ), we assign them to the same type of PL centers but located inside pore walls and on the pore internal surfaces, respectively. The 1.8-eV band efficiency increases when the excitation wavelength changes from 193 to $266 \mathrm{~nm}$, resulting in a shift of the compound's red PL band toward the long wavelength side and in a broadening of the band as a whole (Figures 1-3). From this we conclude that 193-nm light predominantly excites the bulk NBOHCs in MSs, despite the fact that the low-energy band related to surface species becomes quite noticeable for DMSs (Figures 1-3). Alternatively, both the red PL bands contribute to the spectra taken with 266-nm excitation (Figures 1-3). The bimodal structure of the red PL appears more clearly in timeresolved detection mode scans at 300 (Figures 4-9) and $90 \mathrm{~K}$ (Figures 10-15). It can be easily seen that the band related to the bulk NBOHC peaked at $1.93 \mathrm{eV}$, the position of which is in good agreement with that of the NBOHC zero-phonon line 


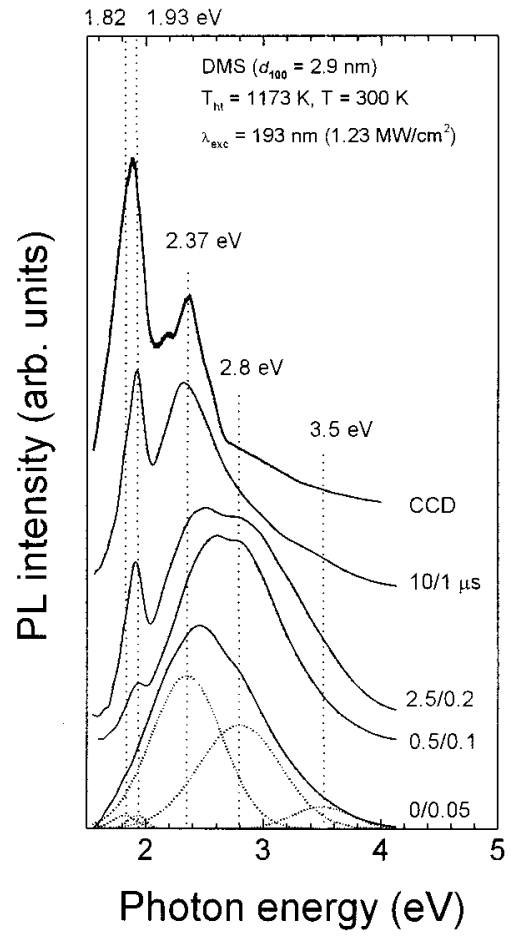

Figure 5. Time-resolved PL spectra measured at $300 \mathrm{~K}$ for DMS $\left(d_{100}\right.$ $=2.9 \mathrm{~nm}$ ) heat-pretreated at $T_{\mathrm{ht}}=1173 \mathrm{~K}$ in comparison to that measured by CCD camera. The spectra were normalized through the blue-green band maximum; $\lambda_{\mathrm{exc}}=193 \mathrm{~nm}, I_{\mathrm{L}}=1.23 \mathrm{MW} / \mathrm{cm}^{2}$. The individual spectrum baselines are arbitrarily shifted for better observation. Numbers at the right side indicate the gate delay and gate width (in microseconds), respectively, at which the corresponding timeresolved spectrum was measured. Dashed lines are Gaussian profiles corresponding to different PL bands. Dashed vertical lines and corresponding numbers in electronvolts show the positions of the Gaussian component peaks. The spectrum measured by CCD camera repeats the corresponding curve in Figure 2.

$(1.935 \pm 0.01 \mathrm{eV})$ observed below $80 \mathrm{~K}$ in glassy $\mathrm{SiO}_{2} \cdot{ }^{15}$ The time decay constant for $1.93-\mathrm{eV}$ emission $(\sim 12 \mu$ s at $300 \mathrm{~K}$ and $\sim 20 \mu$ s at $90 \mathrm{~K}$ ) also agrees with previous data obtained for silica nanoparticles and bulk silica. ${ }^{15,32,33}$ The low-energy red PL band peaked in the range of $1.74-1.84 \mathrm{eV}$ and decays slower $(\sim 50 \mu$ s at $300 \mathrm{~K}$ and $\sim 1.2 \mathrm{~ms}$ at $90 \mathrm{~K})$. This is one reason the band can be detected separately only with a time delay longer than $20 \mu$ s (Figures 13-15).

The blue PL can only be induced by 193-nm light and occurs as a shoulder near the $2.37-\mathrm{eV}$ band in spectra measured in the conventional detection mode (Figures 1-3). However, the band position can be determined more appropriately in time-resolved measurements (Figures 4-6, 10-12). In general, the PL spectra detected by CCD camera correspond to those taken in timeresolved detection mode scans only with time delay longer than $\sim 10$ and $\sim 1 \mu$ s in the case of 193-nm (Figures 4-6, 10-12) and 266-nm excitation (Figures 7-9, 13-15), respectively. It is evident that the CCD camera is an integrated device, so the long time components should contribute to the spectrum with higher efficiency within a given acquisition time. Because the time decay constant of the blue PL $(\sim 5 \mu$ s at $300 \mathrm{~K}$ and $\sim 10$ $\mu \mathrm{s}$ at $\mathrm{T}=90 \mathrm{~K})$ is smaller than those for green $(\sim 15 \mu \mathrm{s}$ at 300 $\mathrm{K}$ and $\sim 40 \mu$ s at $90 \mathrm{~K}$ ) and red emission, the blue band manifests itself with a low amplitude in spectra measured by CCD camera. Similar blue PL induced by $193-\mathrm{nm}$ light has been observed for silica nanoparticles ${ }^{32,33}$ and attributed to STEs..$^{33}$ Note also that the STEPL normally appears for bulk $\mathrm{SiO}_{2} \cdot{ }^{13,19,22,27}$ It is known that the self-trapping of FEs is accompanied by a strong distortion of the $\mathrm{SiO}_{2}$ lattice, which leads to a large Stokes

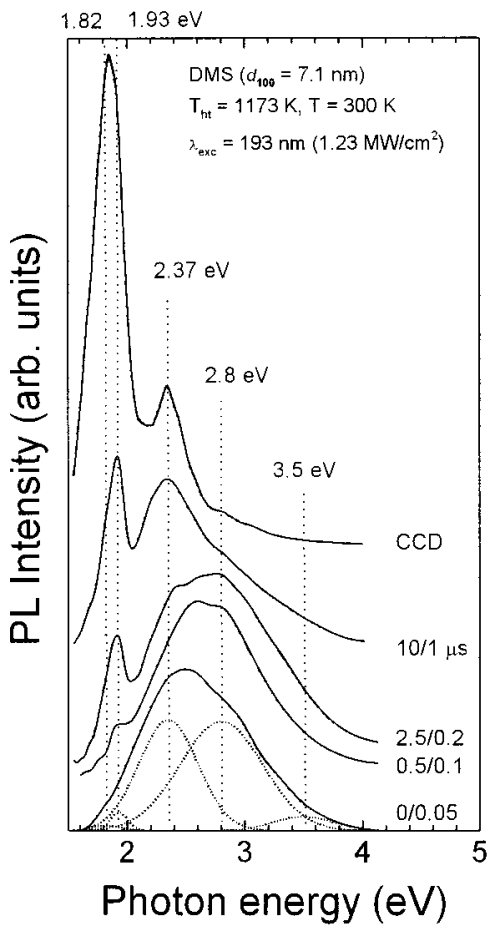

Figure 6. Time-resolved PL spectra measured at $300 \mathrm{~K}$ for DMS $\left(d_{100}\right.$ $=7.1 \mathrm{~nm}$ ) heat-pretreated at $T_{\mathrm{ht}}=1173 \mathrm{~K}$ in comparison to that measured by CCD camera. The spectra were normalized through the blue-green band maximum; $\lambda_{\text {exc }}=193 \mathrm{~nm}, I_{\mathrm{L}}=1.23 \mathrm{MW} / \mathrm{cm}^{2}$. The individual spectrum baselines are arbitrarily shifted for better observation. Numbers at the right side indicate the gate delay and gate width (in microseconds), respectively, at which the corresponding timeresolved spectrum was measured. Dashed lines are Gaussian profiles corresponding to different PL bands. Dashed vertical lines and corresponding numbers in electronvolts show the positions of the Gaussian component peaks. The spectrum measured by CCD camera repeats the corresponding curve in Figure 3.

shift of the band. As a result, intrinsic recombination occurs as a PL band peak at $\sim 2.75 \mathrm{eV}$ with a full width at half-maximum (fwhm) of $\sim 0.7 \mathrm{eV}$ and lifetime $\tau \sim 1 \mathrm{~ms}^{19}$ The emission is originated from a triplet-to-singlet transition in STEs and can be induced by UV light, ${ }^{19}$ energetic electrons, ${ }^{22} \mathrm{X}$-rays, ${ }^{13}$ or MP excitation. ${ }^{27,28,33}$ The most intense STEPL is observed for MCM-41 heat-pretreated at $T_{\mathrm{ht}}=1173 \mathrm{~K}$ (Figure 1). Since the STEs can only be produced in the nanometer-sized bulk fragments of MSs, the mentioned feature reflects the effect of material ordering on the self-trapping process. The STEPL band is peaked at $\sim 2.8 \mathrm{eV}$ (Figures 4-6) with fwhm $\sim 0.65 \mathrm{eV}$, in good agreement with similar parameters for silica nanoparticles ${ }^{33}$ and bulk silica. ${ }^{19}$ The more rapid emission decay in comparison to that typically observed for bulk $\mathrm{SiO}_{2}$ has been suggested to result from an increase in the efficiency of FE self-trapping. ${ }^{33}$ The origin of an UV band peak in the range of $\sim 3.4-3.5 \mathrm{eV}$ (Figures 4-6 and 10-12) remains to be unknown and should be investigated additionally. It becomes evident from the abovementioned reason that since the UV PL decays faster (1-3 (s) than the remaining PL bands, it does not appear in the conventional detection mode scans. Note also that some blue short-time decayed ( $\sim 100 \mathrm{~ns})$ emission also occurs in spectra measured with 266-nm light for the initial heat-untreated DMS of larger size pores (Figure 3). However, this PL disappears completely with the heat pretreatment, so it was attributed to uncontrolled organic impurities.

Since the photon energy of Nd:YAG laser light $(266 \mathrm{~nm}$, $4.66 \mathrm{eV}$ ) coincides very closely with the absorption peak of NBOHCs $(\sim 4.8 \mathrm{eV})^{15}$ compared to ArF laser light $(193 \mathrm{~nm}$, 


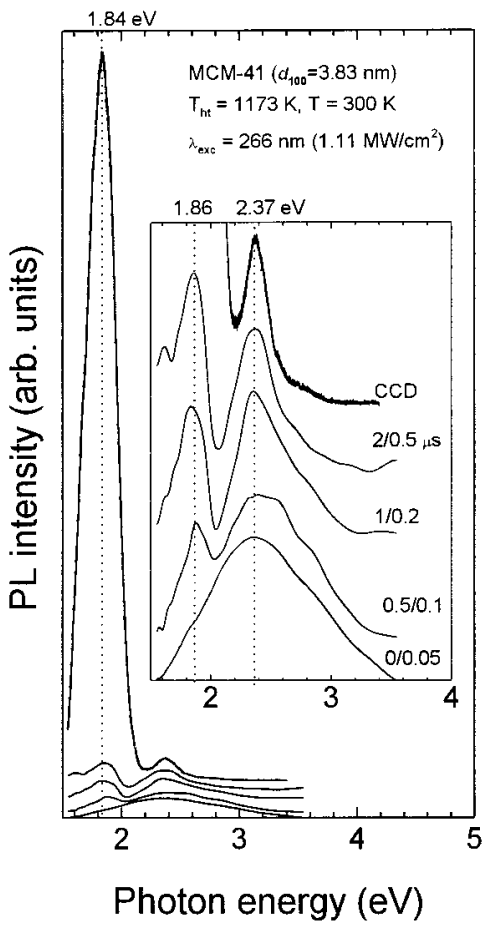

Figure 7. Time-resolved PL spectra measured at $300 \mathrm{~K}$ for MCM-41 $\left(d_{100}=3.83 \mathrm{~nm}\right)$ heat-pretreated at $T_{\mathrm{ht}}=1173 \mathrm{~K}$ in comparison to that measured by CCD camera. The spectra were normalized through the blue-green band maximum; $\lambda_{\mathrm{exc}}=266 \mathrm{~nm}, I_{\mathrm{L}}=1.11 \mathrm{MW} / \mathrm{cm}^{2}$. The inset shows the same PL spectra on an enlarged scale. The individual spectrum baselines are arbitrarily shifted for better observation. Numbers at the right side indicate the gate delay and gate width (in microseconds), respectively, at which the corresponding time-resolved spectrum was measured. Dashed vertical lines and corresponding numbers in electronvolts show the positions of the Gaussian component peaks. The spectrum measured by CCD camera repeats the corresponding curve in Figure 1.

$6.4 \mathrm{eV}$ ), we used 266-nm excitation to probe the effect of 193$\mathrm{nm}$ light on the concentration of NBOHCs. Accordingly, three spectra for each specimen were measured: (1) the spectrum taken with 193-nm excitation; (2) the spectrum taken with 266$\mathrm{nm}$ excitation; (3) the spectrum taken with 266-nm excitation from specimens preirradiated with $193-\mathrm{nm}$ light (30 min). The corresponding curves are shown in Figures 1-3. One can see that the red emission induced by $266-\mathrm{nm}$ light for the preirradiated specimens tends to be weaker, indicating a decrease in the concentration of NBOHCs. However, the preirradiation affects predominantly surface NBOHCs because the resulting red PL peak both decreases in intensity and approaches the position corresponding to bulk NBOHCs $(1.93 \mathrm{eV})$.

The experimental results discussed suggest the different mechanisms of PL excitation with 193- and 266-nm laser light. Similarly, as was proposed for silica nanoparticles, a direct onephoton excitation of intrinsic PL from structural defects and impurities is expected to occur with 266-nm excitation, because the total energy of two laser photons $(9.32 \mathrm{eV})$ is not enough to produce $\mathrm{FEs}$ in $\mathrm{SiO}_{2}(10.2 \mathrm{eV}) .{ }^{13}$ However, this energy would be suitable for direct TP excitation of STEs, which are localized and unable to transfer energy to impurities and structural defects. ${ }^{13}$ Since we did not observe the STEPL induced by 266$\mathrm{nm}$ light, the PL excitation in this case does not involve any excitonic states, so it results from one-photon absorption. Accordingly, the PL originates from bulk NBOHCs and terminal surface $\mathrm{NBOHCs}$ and $\equiv \mathrm{Si}-\mathrm{H}$ when 266-nm light was used.

The second mechanism is associated with an indirect excitation of PL centers through TP-produced FE energy transfer (an

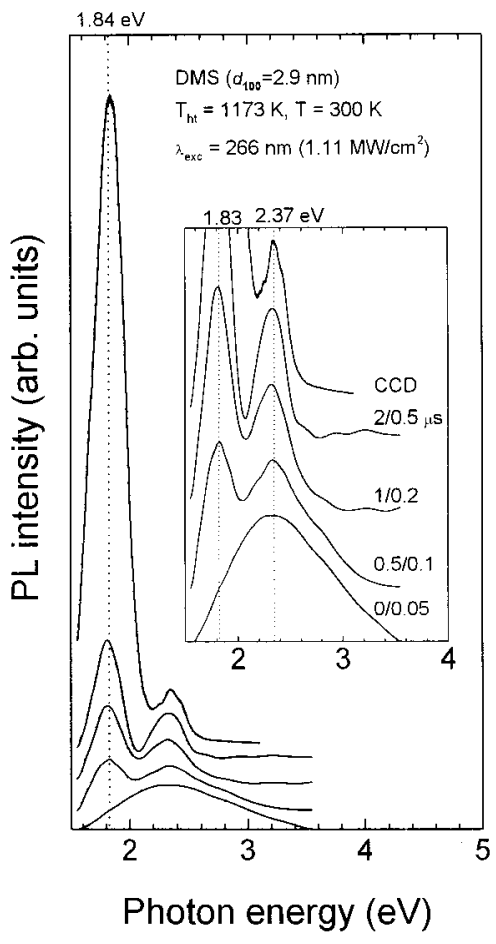

Figure 8. Time-resolved PL spectra measured at $300 \mathrm{~K}$ for DMS $\left(d_{100}\right.$ $=2.9 \mathrm{~nm}$ ) heat-pretreated at $T_{\mathrm{ht}}=1173 \mathrm{~K}$ in comparison to that measured by CCD camera. The spectra were normalized through the blue-green band maximum; $\lambda_{\text {exc }}=266 \mathrm{~nm}, I_{\mathrm{L}}=1.11 \mathrm{MW} / \mathrm{cm}^{2}$. The inset shows the same PL spectra on an enlarged scale. The individual spectrum baselines are arbitrarily shifted for better observation. Numbers at the right side indicate the gate delay and gate width (in microseconds), respectively, at which the corresponding time-resolved spectrum was measured. Dashed vertical lines and corresponding numbers in electronvolts show the positions of the Gaussian component peaks. The spectrum measured by CCD camera repeats the corresponding curve in Figure 2.

inelastic scattering of FEs) followed by excitation of their intrinsic electronic transitions. This happens when 193-nm light was applied (the total energy of two laser photons $12.8 \mathrm{eV}$ ). However, the interaction without energy transfer can occur as well (an elastic scattering). ${ }^{33}$ Therefore, we assume that the FE energy transfer is prevalent for bulk NBOHCs and surface bonded water-like species, but an elastic scattering of FEs is consistent with surface $\mathrm{NBOHC}$ and $\equiv \mathrm{Si}-\mathrm{H}$. As a result, the PL responses originated from bulk NBOHCs and surface-bonded water-like species are only present in spectra measured with 193-nm light. Additionally, the self-trapping of FEs with their subsequent radiative deexcitation is responsible for the STEPL. Hence, in either case the light emission resulted from the relaxation of TP-produced FEs. This is confirmed by the fact that the PL bands related to STEs and bonded water-like species can be induced only simultaneously and exclusively by 193$\mathrm{nm}$ light (Figures 1-3). Because the low-energy red PL band induced by 193-nm light becomes more detectable for DMSs (Figures 2 and 3), we conclude that the material structural peculiarities affect FE energy relaxation in surface species. This is not the case for direct one-photon excitation, for which both the types of NBOHCs can be excited equiprobably. Accordingly, the red PL bands occur in spectra with various intensities for 193- and 266-nm laser sources.

The time decay constant for green PL induced by $266-\mathrm{nm}$ light and assigned to $\equiv \mathrm{Si}-\mathrm{H}$ is smaller compared to that observed for bonded water-like species with 193-nm excitation (Figures 4-9) despite the fact that the PL bands are in practically the same spectral position. This feature can also be understood 


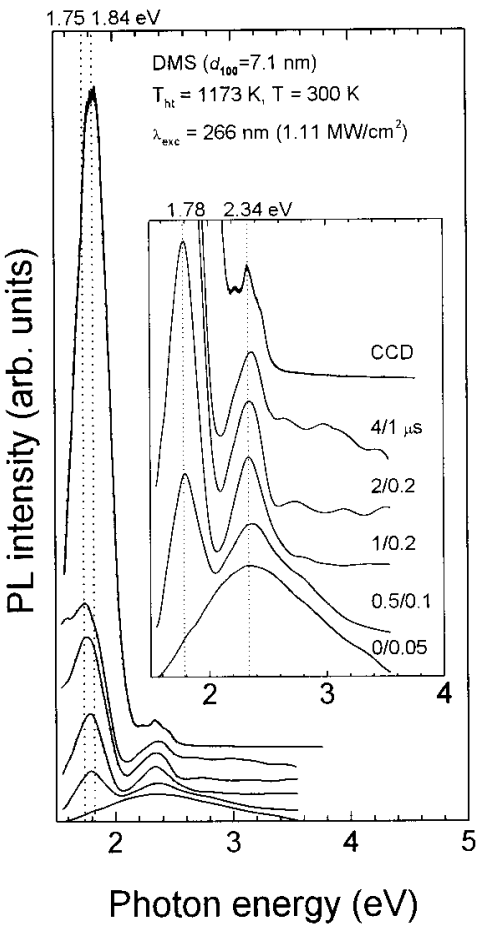

Figure 9. Time-resolved PL spectra measured at $300 \mathrm{~K}$ for DMS $\left(d_{100}\right.$ $=7.1 \mathrm{~nm}$ ) heat-pretreated at $T_{\mathrm{ht}}=1173 \mathrm{~K}$ in comparison to that measured by CCD camera. The spectra were normalized through the blue-green band maximum; $\lambda_{\mathrm{exc}}=266 \mathrm{~nm}, I_{\mathrm{L}}=1.11 \mathrm{MW} / \mathrm{cm}^{2}$. The inset shows the same PL spectra on an enlarged scale. The individual spectrum baselines are arbitrarily shifted for better observation. Numbers at the right side indicate the gate delay and gate width (in microseconds), respectively, at which the corresponding time-resolved spectrum was measured. Dashed vertical lines and corresponding numbers in electronvolts show the positions of the Gaussian component peaks. The spectrum measured by CCD camera repeats the corresponding curve in Figure 3.

in the framework of the model taking into account the different mechanisms of PL excitation. It is evident that the FE energy transfer requires a certain time before PL centers are excited, oppositely to direct one-photon excitation, at which time the PL time decay is determined exclusively by the intrinsic parameters of light emitters. Note also that the extremely high intensity of PL induced by 193-nm light compared to that with 266-nm excitation additionally supports the existence of the two different mechanisms of PL excitation.

It can be easily seen from Figures $4-6$ that there exists a nonmonotonic evolution of the STEPL band with time. The PL intensity rises up in the range of several microseconds beyond the excitation pulse and then decays with a constant of $\sim 5 \mu$ s. Cooling to $90 \mathrm{~K}$ makes the effect faintly detectable (Figures $10-12)$, whereas the time decay becomes longer $(\sim 10 \mu \mathrm{s})$. It is known that the self-trapping process is consistent with a localization of $\mathrm{FEs}$ in $\mathrm{SiO}_{2}$ lattice, which requires a reduction of excitonic energy, giving rise to the STE states inside the energy gap (Figure 16). ${ }^{13}$ It is predicted that there exists a STE barrier for FEs to reach self-trapped states, which can be passed either by tunneling or by activation (Figure 16) ${ }^{42}$ It is reasonable to expect that the FE heating to the energy exceeding the STE activation barrier will enhance self-trapping efficiency. By contrast, FEs are able to reach STE states only by tunneling. It is evident that the FE tunneling from their vibrationally excited states should be very probable. In general, the efficiency of penetration through the barrier increases with the vibrational temperature of FEs and reaches a maximum when the vibrational energy exceeds the STE barrier. It has been proposed recently

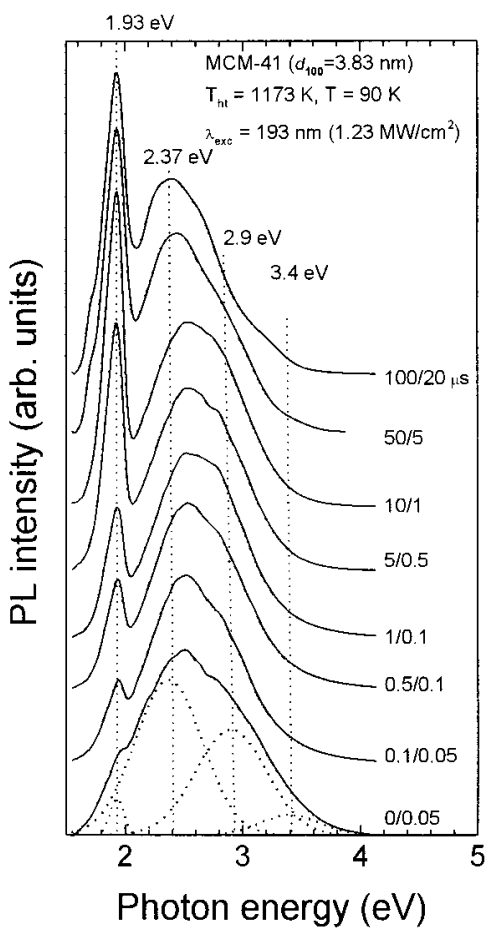

Figure 10. Time-resolved PL spectra measured at $90 \mathrm{~K}$ for MCM-41 $\left(d_{100}=3.83 \mathrm{~nm}\right)$ heat-pretreated at $T_{\mathrm{ht}}=1173 \mathrm{~K}$. The spectra were normalized through the blue-green band maximum; $\lambda_{\text {exc }}=193 \mathrm{~nm}, I_{\mathrm{L}}$ $=1.23 \mathrm{MW} / \mathrm{cm}^{2}$. The individual spectrum baselines are arbitrarily shifted for better observation. Numbers at the right side indicate the gate delay and gate width (in microseconds), respectively, at which the corresponding time-resolved spectrum was measured. Dashed lines are Gaussian profiles corresponding to different PL bands. Dashed vertical lines and corresponding numbers in electronvolts show the positions of the Gaussian component peaks.

that the main specific feature for the laser heating of TPproduced FEs generated in the confined space of silica-based nanoscales is a condition when an intense laser light is applied. ${ }^{33}$ Because the rate of TP transitions is usually small, ${ }^{43}$ much more light power should be used than that required of the one-photon excitation. Additionally, the mean free path length for FEs in bulk silicas is much longer than the size of bulk fragments in MSs (for example, in type III fused silica, $\sim 500 \mathrm{~nm}$ ). ${ }^{13}$ The latter feature means that FEs should suffer collisions with the boundary of confined areas and be scattered by it either elastically or inelastically. Under the mentioned conditions, FEs can gain additional energy from the laser field and be heated to high temperature, creating electron-hole plasma. ${ }^{33}$ It is evident that the frequency of collisions will tend to be higher with decreasing size of bulk fragments. Thus, the PL from MSs induced by 193-nm laser light, from our point of view, reflects the dynamics of FE relaxation in the confined space of nanometer-sized bulk fragments, including elastic and inelastic scattering of FEs by the boundary, the laser heating, and selftrapping processes. The picture suggested resembles the behavior of electron-ion collisions in plasma. ${ }^{44-46}$ The electron heating by electromagnetic waves is well-known, and is usually called the inverse bremsstrahlung process. ${ }^{44,45}$ The mentioned process is associated with the absorption of additional quanta from the laser field due to electron scattering in the presence of a strong electromagnetic wave. The additional energy can be gained either by one-photon absorption or by the MP process, depending on the intensity and frequency of laser light. ${ }^{44-46}$ Because the nonlinear inverse bremsstrahlung process is expected to occur at laser light intensity much higher than that used in our measurements, we consider here only the linear FE laser heating. 


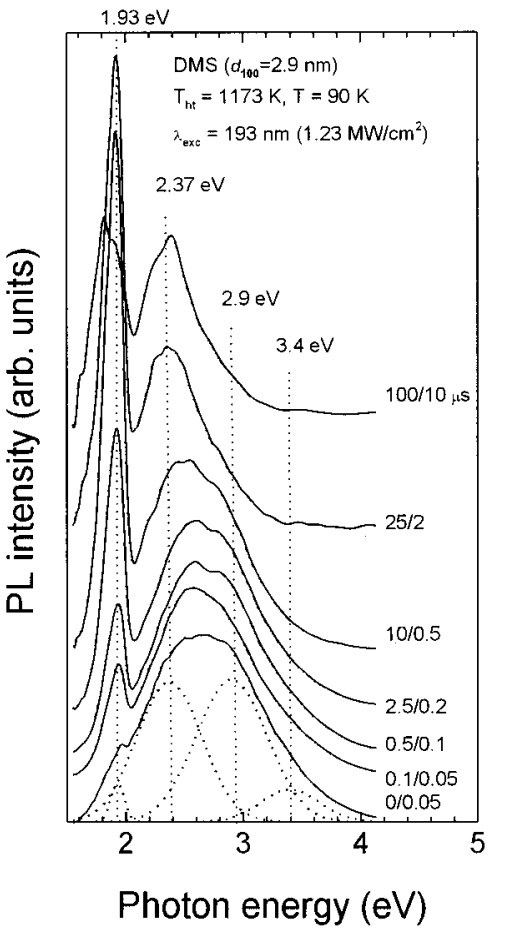

Figure 11. Time-resolved PL spectra measured at $90 \mathrm{~K}$ for DMS $\left(d_{100}\right.$ $=2.9 \mathrm{~nm}$ ) heat-pretreated at $T_{\mathrm{ht}}=1173 \mathrm{~K}$. The spectra were normalized through the blue-green band maximum; $\lambda_{\mathrm{exc}}=193 \mathrm{~nm}, I_{\mathrm{L}}=1.23 \mathrm{MW} /$ $\mathrm{cm}^{2}$. The individual spectrum baselines are arbitrarily shifted for better observation. Numbers at the right side indicate the gate delay and gate width (in microseconds), respectively, at which the corresponding timeresolved spectrum was measured. Dashed lines are Gaussian profiles corresponding to different PL bands. Dashed vertical lines and corresponding numbers in electronvolts show the positions of the Gaussian component peaks.

Accordingly, the maximal temperature of laser-heated FEs can be estimated using the gas kinetics approximation ${ }^{46}$ as follows: $T_{\max }=(2 / 3)(6.4 \mathrm{eV} / k) \approx 0.5 \times 10^{5} \mathrm{~K}$, where $k$ is the Boltzmann constant. The obtained value is typical for MP-produced excitons ${ }^{47}$ and laser-heated plasma. ${ }^{46}$

The temperature at which the spectra of specimens have been measured will affect the efficiency of FE laser heating and in turn the FE pass through the STE barrier, because of the factor $k T$. It is known that the $\mathrm{FE}$ motion in $\mathrm{SiO}_{2}$ lattice occurs as thermally activated hops with activation energy $\sim 0.1 \mathrm{eV} .{ }^{13}$ Because the FE energy taken initially within the FE generation process is assumed to be smaller than the STE activation barrier, the temperature of specimens strongly affects the FE hopping, preceding collisions with the boundary of nanometer-sized bulk fragments. However, if the FE reaches the boundary and elasticaly scatters by it in the presence of an intense laser field, the FE energy increases significantly to the value exceeding the STE barrier. Hence, one can conclude that the nonmonotonic evolution of the STEPL response with time could be associated with the laser heating of FEs. Since the latter process takes place only when FE reaches the boundary and since the thermally activated motion of FEs before collisions with the boundary requires a certain time, the maximum of the STEPL occurs in the range of several microseconds beyond the excitation pulse. If the temperature decreases to $90 \mathrm{~K}$, the FE laser-heating efficiency becomes lower because the FE hopping is hampered and only a small number of FEs can reach the boundary. As a result, the FE is able to pass through the STE barrier dominantly by tunneling and the mentioned feature disappears completely. The conception of FE laser heating can also explain the shorter time decay of the STEPL from MSs in comparison with that

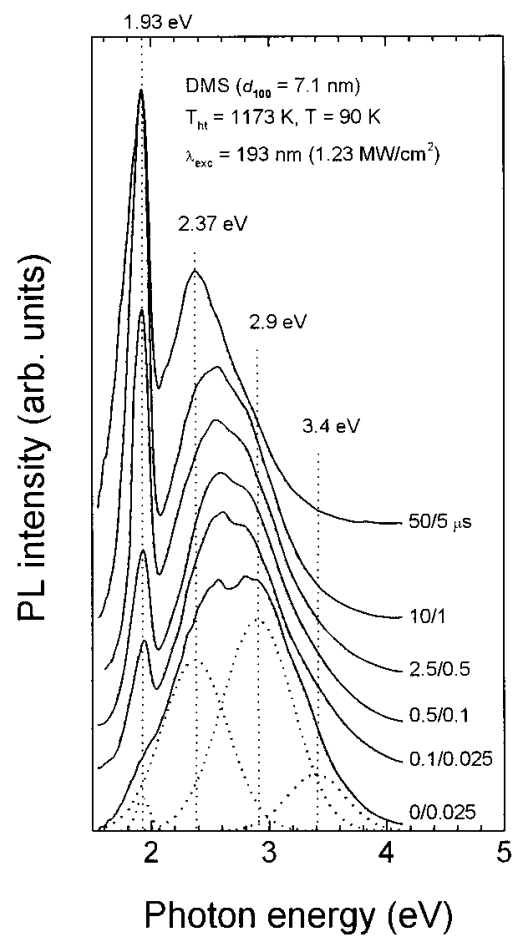

Figure 12. Time-resolved PL spectra measured at $90 \mathrm{~K}$ for DMS $\left(d_{100}\right.$ $=7.1 \mathrm{~nm}$ ) heat-pretreated at $T_{\mathrm{ht}}=1173 \mathrm{~K}$. The spectra were and normalized through the blue-green band maximum; $\lambda_{\text {exc }}=193 \mathrm{~nm}, I_{\mathrm{L}}$ $=1.23 \mathrm{MW} / \mathrm{cm}^{2}$. The individual spectrum baselines are arbitrarily shifted for better observation. Numbers at the right side indicate the gate delay and gate width (in microseconds), respectively, at which the corresponding time-resolved spectrum was measured. Dashed lines are Gaussian profiles corresponding to different PL bands. Dashed vertical lines and corresponding numbers in electronvolts show the positions of the Gaussian component peaks.

from bulk silica, because of an increase in the efficiency of the STE barrier penetration for laser-heated FEs. ${ }^{33}$ This conclusion takes into account the fact that the total time decay constant consists of two components resulting from FE penetration through the STE barrier and intrinsic lifetime for STEs.

Since the 193-nm light irradiation reduces the concentration of surface NBOHCs, we assume that additionally to the FEs elastic scattering by these surface species, the inelastic scattering can also occur, leading to NBOHC breakdown with oxygen desorption. It is known that the $\mathrm{FE}$ formation in $\mathrm{SiO}_{2}$ is due to the displacement of one of the four oxygens of $\mathrm{SiO}_{4}$ tetrahedron from its equilibrium position. ${ }^{13}$ If the interaction of this excitation with the terminal surface $\mathrm{NBOHC}$ occurs inelastically, the activation of oxygen motion along the surface takes place. However, this is not the case for bulk NBOHCs because the oxygen motion is hampered by the $\mathrm{SiO}_{2}$ lattice. Since in this case the oxygen remains localized nearby the silicon site and can be trapped back, the excitation process is assumed to be a simple transition into the excited state. If this happens on the surface, the mobile oxygen can move away, breaking the NBOHC as a whole. Note that the oxygen mobility on the surface can also be hampered by structural peculiarities, and hence, the surface NBOHCs will be able to take part in the light emission process as well. Since the oxygen motion is assumed to be along pores, the surface NBOHC breakdown is more efficient for ordered MSs. In DMSs the surface species can remain stable and hence gently contribute to the PL spectrum with 193-nm excitation (Figures 2, 3, 11, and 12).

We propose a similar situation to occur with $\equiv \mathrm{Si}-\mathrm{H}$ species. The FE energy relaxation in this surface center activates the hydrogen motion. However, if the motion of mobile hydrogen 


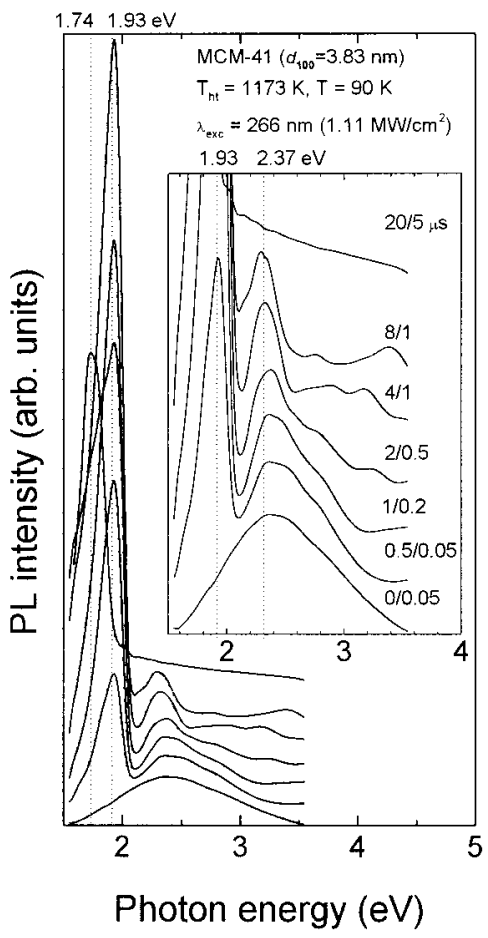

Figure 13. Time-resolved PL spectra measured at $90 \mathrm{~K}$ for MCM-41 $\left(d_{100}=3.83 \mathrm{~nm}\right)$ heat-pretreated at $T_{\mathrm{ht}}=1173 \mathrm{~K}$. The spectra were normalized at the $2.37-\mathrm{eV}$ band intensity; $\lambda_{\mathrm{exc}}=266 \mathrm{~nm}, I_{\mathrm{L}}=1.11$ $\mathrm{MW} / \mathrm{cm}^{2}$. The inset shows the same PL spectra on an enlarged scale. The individual spectrum baselines are arbitrarily shifted for better observation. Numbers at the right side indicate the gate delay and gate width (in microseconds), respectively, at which the corresponding timeresolved spectrum was measured. Dashed vertical lines and corresponding numbers in electronvolts show the positions of the Gaussian component peaks.

is limited for various reasons, hydrogen atoms can be trapped back to the silicon site with subsequent emission in a similar manner as directly excited hydrogen-related species. This condition is expected to be realized if the surface is covered by adsorbed water (the initial specimen) or if $\equiv \mathrm{Si}-\mathrm{O}^{\bullet}$ and $\equiv \mathrm{Si}-\mathrm{H}$ species form bonded water-like species (the heat-pretreated specimens). The mentioned assumption explains the similarity of PL spectra induced by 193- and 266-nm light for initial heatuntreated specimens (Figures 1-3). Thus, the FE collision with the boundary is suggested to occur as either elastic or inelastic scattering. The energy transfer due to an inelastic scattering of FEs can involve the terminal surface atom desorption, breaking the surface species as a whole. Under specific conditions the mobile atoms can be trapped back on $\equiv \mathrm{Si}^{\bullet}$ sites and further energy relaxation leads to light emission. The model proposed allows us to explain all spectroscopic features observed, but the ultimate answer to the problem discussed can be found within further experimental and theoretical investigations.

Another type of excitation is realized with 266-nm light. According to the model developed for bulk NBOHC, ${ }^{15}$ the excitation is attributed to a transition from the $\equiv \mathrm{Si}-\mathrm{O}^{*}$ $\sigma$-bonding orbital to the $2 \mathrm{p}$ nonbonding orbital of nonbridging oxygen. The PL is due to the charge transfer transition between the singly occupied nonbonding $2 p \pi$-orbital of nonbridging oxygen and the lone-pair $2 p$ orbital of one of the nearest bridging oxygens. This model explains the relatively long PL lifetime (about $20 \mu \mathrm{s}$ ). The electron transition onto the nonbonding orbital only makes the $\mathrm{Si}-\mathrm{O}$ bond weaker, but the oxygen is unable to move from its equilibrium position. The excitation of hydrogen-related species is assumed to be similar. However, the excitation energy of $\equiv \mathrm{Si}-\mathrm{H}$ centers is much smaller

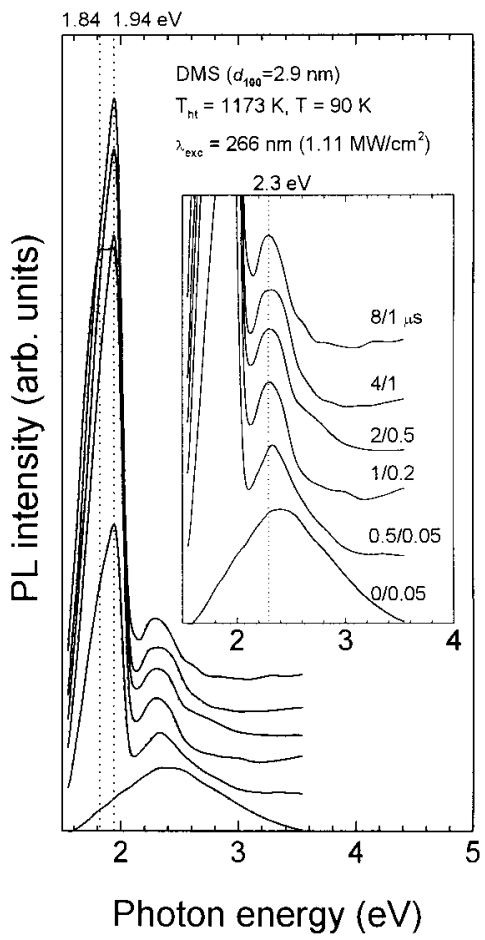

Figure 14. Time-resolved PL spectra measured at $90 \mathrm{~K}$ for DMS $\left(d_{100}\right.$ $=2.9 \mathrm{~nm}$ ) heat-pretreated at $T_{\mathrm{ht}}=1173 \mathrm{~K}$. The spectra were normalized at the $2.37-\mathrm{eV}$ band intensity; $\lambda_{\mathrm{exc}}=266 \mathrm{~nm}, I_{\mathrm{L}}=1.11 \mathrm{MW} / \mathrm{cm}^{2}$. The inset shows the same PL spectra on an enlarged scale. The individual spectrum baselines are arbitrarily shifted for better observation. Numbers at the right side indicate the gate delay and gate width (in microseconds), respectively, at which the corresponding time-resolved spectrum was measured. Dashed vertical lines and corresponding numbers in electronvolts show the positions of the Gaussian component peaks.

compared to that required of nonbonding state excitation $(\sim 8$ $\mathrm{eV}){ }^{21}$ This fact indicates that hydrogens are involved in a more complicated surface site than a simple $\equiv \mathrm{Si}^{\bullet}$ dangling bond. In any case, the 266-nm excitation energy is not enough to initiate the hydrogen motion.

The above-mentioned two mechanisms of PL excitation are also manifested in the IDPLY measurements for the discussed PL bands (Figures 17-19). The IDPLY obtained with 266-nm excitation is linear. It is known that the $\alpha$-photon excitation rate ( $\alpha$ denotes the number of photons required of intrinsic PL excitation) can be presented as follows: ${ }^{43} W^{(\alpha)}=\sigma^{(\alpha)} I_{L}^{\alpha}$, where $\sigma^{(\alpha)}$ is the cross section of the $\alpha$-photon process and $I_{\mathrm{L}}$ is the laser light intensity. Such power-law dependences are typical for MP-excited PL, $, 27,28,33,44$ because the PL intensity is proportional to the MP excitation rate. ${ }^{43}$ The usual way to analyze the IDPLY is to draw it in the $\log -\log$ scale. Then the resulting slope will show the photonicity of the excitation process. Therefore, the linearity of IDPLY indicates a one-photon process of PL excitation (Figures 17-19). As this takes place, the heat pretreatment does not affect the IDPLY slopes. However, a saturation of the PL intensity sometimes occurs with most power laser light.

The situation is considerably different with 193-nm excitation. The dynamics of PL excitation is much complicated in this case. There exist several inflection points, after which the slope changes. The IDPLY slope for MCM-41 taken within quite small laser light intensities is $\sim 1$ for the initial and heatpretreated at $T_{\mathrm{ht}}=873 \mathrm{~K}$ specimens, but it becomes $\alpha=2$ with increasing $T_{\text {ht }}$ (Figure 17). As a final stage, which can be reached at stronger light, the IDPLY slopes change, normally 


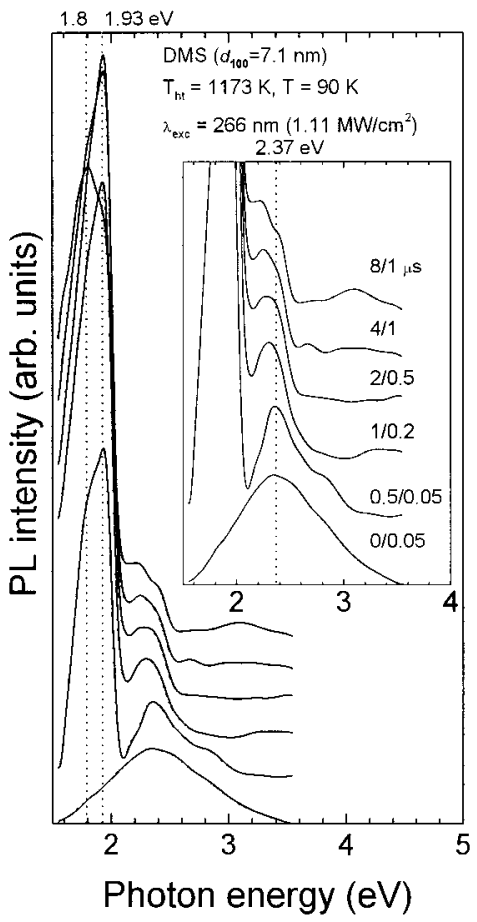

Figure 15. Time-resolved PL spectra measured at $90 \mathrm{~K}$ for DMS $\left(d_{100}\right.$ $=7.1 \mathrm{~nm}$ ) heat-pretreated at $T_{\mathrm{ht}}=1173 \mathrm{~K}$. The spectra were normalized at the $2.37-\mathrm{eV}$ band intensity; $\lambda_{\mathrm{exc}}=266 \mathrm{~nm}, I_{\mathrm{L}}=1.11 \mathrm{MW} / \mathrm{cm}^{2}$. The inset shows the same PL spectra on an enlarged scale. The individual spectrum baselines are arbitrarily shifted for better observation. Numbers at the right side indicate the gate delay and gate width (in microseconds), respectively, at which the corresponding time-resolved spectrum was measured. Dashed vertical lines and corresponding numbers in electronvolts show the positions of the Gaussian component peaks.

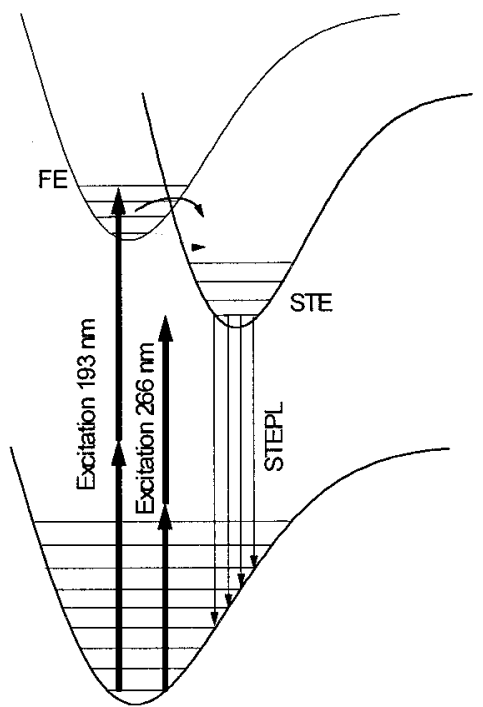

Figure 16. Schematic diagram in the terms of chemical bonding in $\equiv \mathrm{Si}-\mathrm{O}-\mathrm{Si} \equiv$ clusters for the self-trapping process of FE in silica and corresponding absorption and emission transitions. The solid and dashed arrows show the two possibilities of the STE barrier penetration by activation or by tunneling, respectively.

approaching a value less than that for the initial stage. This fact is likely to indicate that the IDPLY slope is affected by the concentration of NBOHCs on the pore internal surface of MCM41, which tends to be larger with increasing $T_{\mathrm{ht}}$ (Figures 1-3).

The IDPLY slope remains to be close to linear within relatively low laser light intensities for small pore sized DMSs heat-pretreated at various $T_{\text {ht }}$ (Figure 18). However, it increases

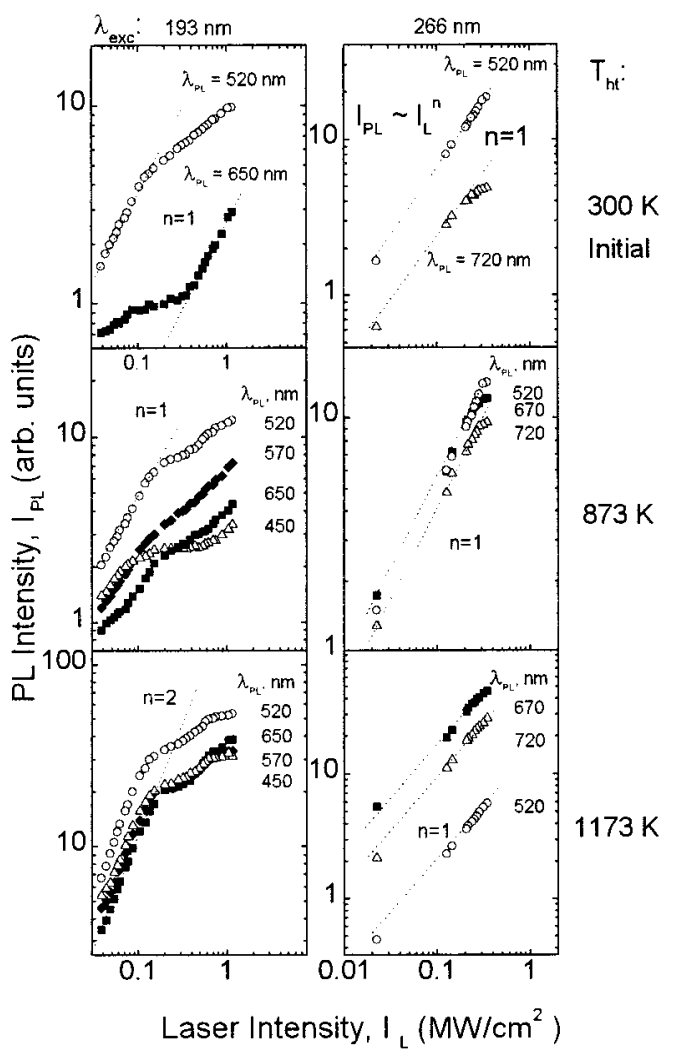

Figure 17. IDPLY observed for $300 \mathrm{~K}$ PL bands for MCM-41 $\left(d_{100}\right.$ $=3.83 \mathrm{~nm}$ ) heat-pretreated at various temperature $\left(T_{\mathrm{ht}}\right)$ and measured with $\lambda_{\mathrm{exc}}=193$ and $266 \mathrm{~nm}$. $\lambda_{\mathrm{PL}}$ denotes the wavelength in the spectrum at which the corresponding IDPLY has been measured. Dashed lines are drawn as a guide to the eye showing the different indices of power function $I_{\mathrm{PL}} \sim I_{\mathrm{L}}{ }^{n}$ in $\log -\log$ scale.

up to almost $\alpha=2$ for heat-pretreated specimens in the case of larger pore size material (Figure 19). The further increase in the laser light intensity brings into existence the inflection points. These data indicate that the IDPLY slope does not reflect directly the photonicity of the absorption process. Because of the indirect mechanism of PL excitation, the IDPLY slopes are affected by the pathways of FE energy relaxation. We conclude that the IDPLY reflects the dynamic of FE relaxation in the confined space of the nanometer-sized bulk fragments of MSs. This dynamic includes several processes, such as laser heating of TP-produced FEs, elastic and inelastic scattering by the boundary of the confined areas, and the interaction of FEs to one another. We predict that one can reach the condition of high-density nonequilibrium electron-hole plasma in MSs by applying 193-nm ArF laser excitation. As a result, the biexcitonic states can be formed with subsequent nonradiative relaxation into the structural defects. ${ }^{26}$ Because such phenomena were observed for silica nanoparticles, ${ }^{33}$ one can conclude that the FE dynamics in MSs includes the same relaxation processes. Extremely high PL efficiency observed with 193-nm excitation as compared to that with 266-nm additionally supports the unconventional mechanism of PL excitation, which most likely reflects the FE density saturation in silica-based nanoscales.

Note that the PL induced from MSs is very similar to those from surface-oxidized silicon nanocrystals ${ }^{34}$ and porous silicon, ${ }^{35-40}$ indicating that the nature of light emitters is the same. However, the comparative analysis of the PL properties of MSs and silicon nanoscales requires a separate consideration and lies beyond the scope of the current paper. 


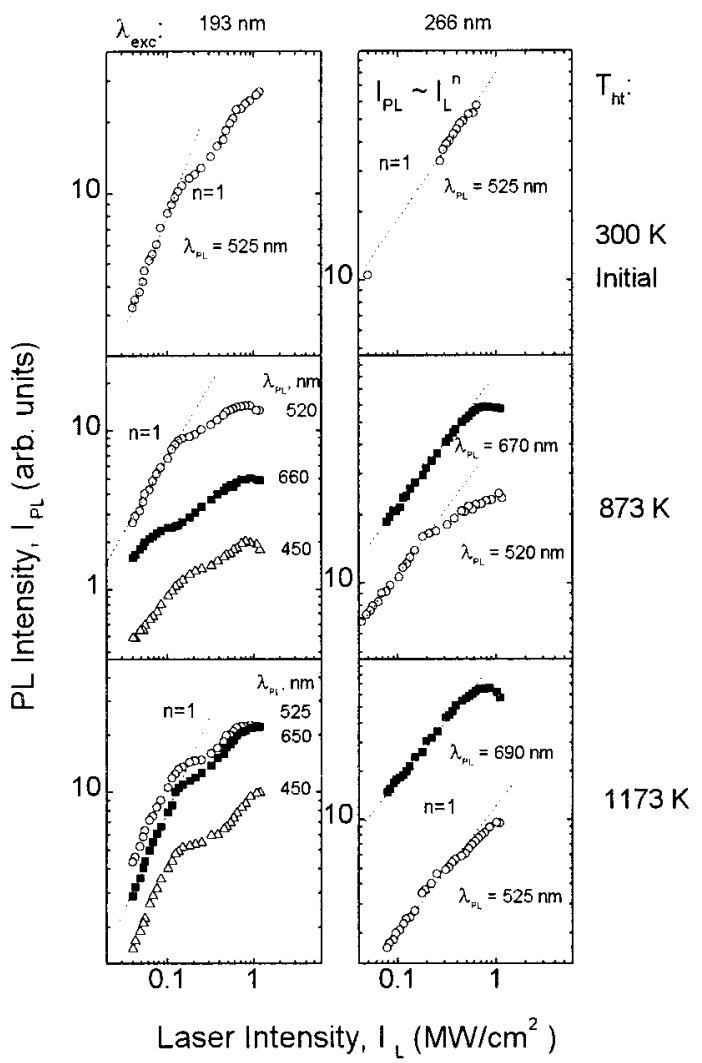

Figure 18. IDPLY observed for $300 \mathrm{~K}$ PL bands for DMS $\left(d_{100}=\right.$ $2.9 \mathrm{~nm})$ heat-pretreated at various temperature $\left(T_{\mathrm{ht}}\right)$ and measured with $\lambda_{\text {exc }}=193$ and $266 \mathrm{~nm}$. $\lambda_{\mathrm{PL}}$ denotes the wavelength in the spectrum at which the corresponding IDPLY has been measured. Dashed lines are drawn as a guide to the eye showing the different indices of power function $I_{\mathrm{PL}} \sim I_{\mathrm{L}}{ }^{n}$ in $\log -\log$ scale.

\section{Conclusions}

We showed that the PL spectra for MCM-41 and DMSs are similar to those for silica nanoparticles. The PL centers are common to both the nanoscale materials. The heat pretreatment of specimens in air results in a rearrangement of the pore internal surfaces, leading to the formation of bonded water-like species and NBOHCs. The PL is originated from the hydrogen-related species and NBOHCs. Additionally, there exists an emission resulting from radiative STE relaxation. The PL spectra measured with 266- and 193-nm excitations are somewhat different because of the different mechanisms of excitation: direct one-photon and indirect through the TP-produced FEs, respectively. The FE energy transfer to PL centers is proposed to occur as an alternative process to self-trapping.

The main difference in the PL properties between MSs and bulk materials is suggested to result from the specific behavior of FEs in the confined space of nanometer-sized bulk fragments. The laser heating of FEs due to their elastic scattering by the boundary in the presence of an intense laser field is suggested to be an important factor in the enhancement of FE self-trapping efficiency. The maximal intensity of STEPL was observed for MCM-41, indicating the influence of material ordering on the self-trapping process. The extremely high PL efficiency is suggested to result from high-density electron-hole plasma produced by ArF laser light in nanometer-sized bulk fragments.

Acknowledgment. The authors acknowledge Academia Sinica and China Petroleum Corp. of Taiwan, Republic of China, for financial support. The work also was supported by National

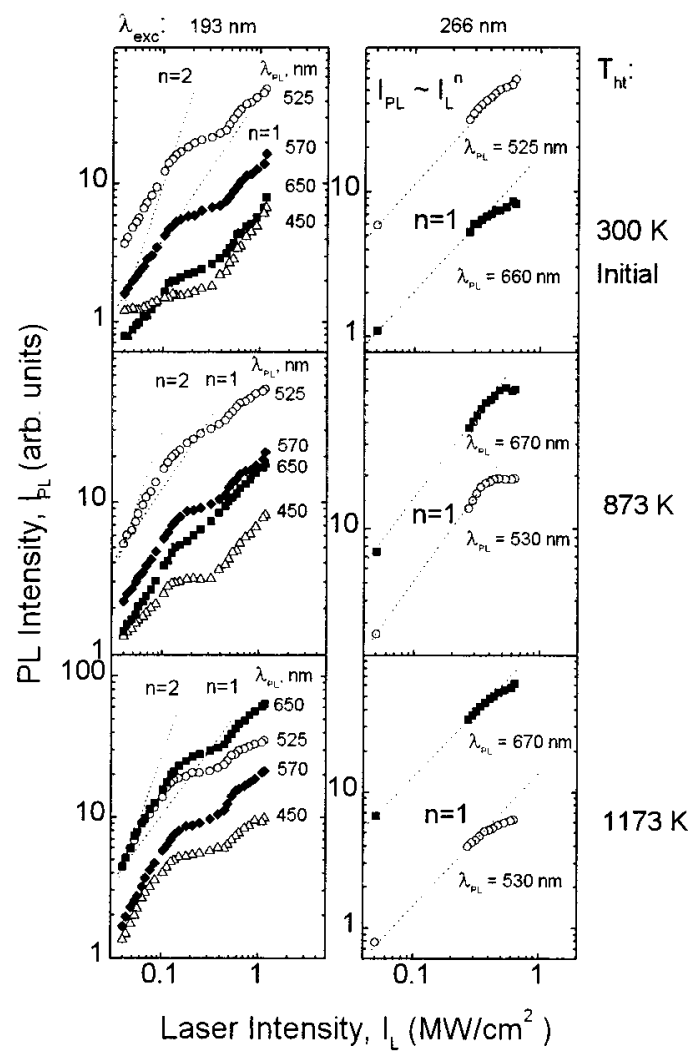

Figure 19. IDPLY observed for $300 \mathrm{~K}$ PL bands for DMS $\left(d_{100}=\right.$ $7.1 \mathrm{~nm})$ heat-pretreated at various temperature $\left(T_{\mathrm{ht}}\right)$ and measured with $\lambda_{\text {exc }}=193$ and $266 \mathrm{~nm}$. $\lambda_{\mathrm{PL}}$ denotes the wavelength in the spectrum at which the corresponding IDPLY has been measured. Dashed lines are drawn as a guide to the eye showing the different indices of power function $I_{\mathrm{PL}} \sim I_{\mathrm{L}}{ }^{n}$ in $\log -\log$ scale.

Science Council of Taiwan: Y.D.G. and S.H.L., Grant No 892113-M-001-050; L.P.H., Grant No 89-2113-M-002-033; Y.T.C., Grant No 89-2113-M-001-032.

\section{References and Notes}

(1) Kresge, C. T.; Leonowicz, M. E.; Roth, W. J.; Vartuli, J. C.; Beck, J. S. Nature 1992, 359, 710-715; Monnier, A.; Schuth, F.; Huo, Q.; Kumar, D.; Margolese, D.; Maxwell, R. S.; Stucky, G. D.; Krishnamurty, M.; Retroff, P.; Firouzi, A.; Janicke, M.; Chmelka, B. F. Science 1993, 261, $1299-1303$

(2) Schmidt, R.; Hansen, E. W.; Stocker, M.; Akporiaye, D.; Ellestad, O. H. J. Am. Chem. Soc. 1995, 117, 4049-4056. 8835 .

(3) Glinka, Yu. D.; Jaroniec, M. J. Phys. Chem. B 1997, 101, 8832-

(4) Glinka, Yu. D.; Jaroniec, C. P.; Jaroniec, M. J. Colloid Interface Sci. 1998, 201, 210-219.

(5) Glinka, Yu. D. J. Exp. Theor. Phys. (Eng. Transl.) 1997, 84, 957970.

(6) El-Shall, M. S.; Li, S.; Turkki, T.; Graiver, D.; Pernisz, U. C., Baraton, M. I. J. Phys. Chem. B 1995, 99, 17805-17809.

(7) Thess, A.; Lee, R.; Nikolaev, P.; Dai, H.; Petit, P.; Robert, J.; Xu, C.; Lee, Y. H.; Kim, S. G.; Rinzler, A. G.; Colbert, D. T.; Scuseria, G. E.; Tomanek, D.; Fischer, J. E.; Smalley, R. E. Science 1996, 273, 483-487.

(8) Journer, C.; Maser, W. K.; Bernier, P.; Loiseau, A.; Lamy de la Chapelle, M.; Lefrant, S.; Deniard, P.; Lee, R.; Fischer, J. E. Nature 1997, $388,756-758$

(9) Zhao, X. S.; Lu, G. Q. J. Phys. Chem. B 1998, 102, 1556-1561.

(10) Ulagappan, N.; Rao, C. N. R. Chem. Commun. 1996, 2759-2760.

(11) Jentys, A.; Pham, N. H.; Vinek, H. J. Chem. Soc., Faraday Trans. 1996, 92, 3287-3291.

(12) Jentys, A.; Kleestorfer, K.; Vinek, H. Micropor. Mesopor. Mater. 1999, 27, 321-328

(13) Trukhin, A. N. J. Non-Cryst. Solids 1992, 149, 32-45.

(14) Griscom, D. L. J. Ceramic Soc. Jpn. 1991, 99, 923-942.

(15) Skuja, L. J. Non-Cryst. Solids 1994, 179, 51-69.

(16) Skuja, L. J. Non-Cryst. Solids 1998, 239, 16-48. 
(17) Hosono, H.; Kawazoe, H.; Matsunami, N. Phys. Rev. Lett. 1998, $80,317-320$

(18) Skuja, L.; Guttler, B. Phys. Rev. Lett. 1996, 77, 2093-2096.

(19) Itoh, C.; Tanimura, K.; Itoh, M.; Itoh, N. Phys. Rev. B 1989, 39 , 11183-11186.

(20) Griscom, D. L.; Mizuguchi, M. J. Non-Cryst. Solids 1998, 239, 66-77.

(21) Pacchioni, G.; Ierano, G. Phys. Rev. B 1998, 57, 818-832.

(22) Stevens Kalceff, M. A. Phys. Rev. B 1998, 57, 5674-5683.

(23) Imai, H.; Arai, K.; Hosono, H.; Abe, Y.; Arai, T.; Imagawa, H. Phys. Rev. B 1991, 44, 4812-4818.

(24) Arai, K.; Imai, H.; Hosono, H.; Abe, Y.; Imagawa, H. Appl. Phys. Lett. 1988, 53, 1891-1893.

(25) Nishikawa, H.; Nakamura, R.; Tohmon, R.; Ohki, Y.; Sakurai, Y.; Nagasawa, K.; Hama Y. Phys. Rev. B 1990, 41, 7828-7834.

(26) Tsai, T. E.; Griscom, D. L. Phys. Rev. Lett. 1991, 67, 2517-2520.

(27) Joosen, W.; Guizard, S.; Martin, P.; Petite, G.; Agostini, P.; Dos Santos, A.; Grillon, G.; Hulin, D.; Migus, A.; Antonetti, A. Appl. Phys. Lett. 1992, 61, 2260-2262.

(28) Glinka, Yu. D.; Naumenko, S. N.; Degoda, V. Ya. J. Non-Cryst. Solids 1993, 152, 219-224.

(29) Goldberg, M.; Fitting, H.-J.; Trukhin, A. J. Non-Cryst. Solids 1997, 220, 69-77.

(30) Trukhin, A. N.; Goldberg, M.; Jansons, J.; Fitting, H.-J.; Tale, I. A. J. Non-Cryst. Solids 1998, 223, 114-122.

(31) Prokes, S. M.; Carlos, W. E.; Veprek, S.; Ossadnic, Ch. Phys. Rev. $B$ 1998, 58, 15632-15635.

(32) Glinka, Yu. D.; Lin, S. H.; Chen, Y.-T. Appl. Phys. Lett. 1999, 75, $778-780$
(33) Glinka, Yu. D.; Lin, S. H.; Chen, Y.-T. Phys. Rev. B 2000, 62, 4733-4743.

(34) Brus, L. E.; Szajowski, P. F.; Wilson, W. L., Harris, T. D.; Schuppler, S.; Citrin, P. H. J. Am. Chem. Soc. 1995, 117, 2915-2922.

(35) Cooke, D. W.; Bennett, B. L.; Farnum, E. H.; Hults, W. L.; Sickafus, K. E.; Smith, J. F.; Taylor, T. N.; Tiwari, P. Appl. Phys. Lett. 1996, 68, $1663-1665$.

(36) Zhu, M.; Han, Y.; Wehrspohn, R. B.; Godet, C.; Etemadi, R.; Ballutaud, D. J. Appl. Phys. 1998, 83, 5386-5393.

(37) Yuan, J.; Haneman, D.; Andrienko, I.; Li, W. J. Appl. Phys. 1998, $83,4385-4388$

(38) Prokes, S. M.; Glembocki, O. J. Phys. Rev. B 1994, 49, $2238-$ 2241.

(39) Gole, J. L.; Dixon, D. A. J. Phys. Chem. B 1998, 102, 33-39.

(40) Gole, J. L.; Dudel, F. P.; Grantier, D.; Dixon, D. A. Phys. Rev. B 1997, 56, 2137-2153.

(41) Herzberg, G. Molecular Spectra and Molecular Structure: Infrared and Raman Spectra of Polyatomic Molecules; Krieger: New York, 1991

(42) Rashba E. I. In Excitons; Rashba, E. I., Sturge, M. D., Eds.; Elsevier: Amsterdam, 1987; pp 273-332.

(43) Shen, Y. R. The Principles of Nonlinear Optics; Wiley: New York, 1984.

(44) Bunkin, F. B.; Kazakov, A. E.; Fedorov M. V. Sov. Physics Uspekhi 1973, 15, 416-435

(45) Kroll, N. M.; Watson, K. M. Phys. Rev. A 1973, 8, 804-809.

(46) Koroteev, N. I.; Shumay, I. L. Physics of High-Power Laser Radiation; Nauka Publishers: Moscow, 1991 (in Russian).

(47) Glinka, Yu. D.; Lin, K.-W.; Chang, H.-C.; Lin, S. H. J. Phys. Chem. B 1999, 103, 4251-4263. 